\title{
Therapeutic effect of transplantation of human bone marrow-derived mesenchymal stem cells on neuron regeneration in a rat model of middle cerebral artery occlusion
}

\author{
PING XIE $^{1 *}$, MING DENG ${ }^{2 *}$, QIN-GUO SUN ${ }^{1}$, YONG-GANG MA $^{2}$, YAN ZHOU ${ }^{2}$, \\ JIANG-HUA MING ${ }^{2}$, QING CHEN ${ }^{2}$, SHI-QING LIU ${ }^{2}$, JUN-QI LIU ${ }^{3}, \mathrm{JUN} \mathrm{CAI}^{4}$ and FEI WU ${ }^{2}$ \\ ${ }^{1}$ Department of Chinese Traditional Medicine, Tongren Hospital of Wuhan University (Wuhan Third Hospital); \\ ${ }^{2}$ Department of Orthopedics, Renmin Hospital of Wuhan University, Wuhan, Hubei 430060; \\ ${ }^{3}$ Department of Radiation Oncology, The First of Affiliated Hospital of Zhengzhou University, Zhengzhou, Henan 45003; \\ ${ }^{4}$ Department of Emergency and Trauma Surgery, The Central Hospital of Wuhan, Tongji Medical College, \\ Huazhong University of Science and Technology, Wuhan, Hubei 430014, P.R. China
}

Received July 5, 2018; Accepted May 31, 2019

DOI: $10.3892 / \mathrm{mmr} .2019 .10536$

\begin{abstract}
Human bone marrow-derived mesenchymal stromal cells (hBMSCs) have been revealed to be beneficial for the regeneration of tissues and cells in several diseases. The present study aimed to elucidate the mechanisms underlying the effect of hBMSC transplantation on neuron regeneration in a rat model of middle cerebral artery occlusion (MCAO). The hBMSCs were isolated, cultured and identified. A rat model of MCAO was induced via the modified Longa method. Neurological severity scores (NSS) were adopted for the evaluation of neuronal function in the model rats after cell transplantation. Next, the expression levels of nestin, $\beta$-III-tubulin ( $\beta$-III-Tub), glial fibrillary acidic protein (GFAP), HNA and neuronal nuclear antigen (NeuN) were examined, as well as the positive expression rates of human neutrophil alloantigen (HNA), nestin, NeuN, $\beta$-III-Tub and GFAP. The NSS, as well as the mRNA and protein expression of nestin, decreased at the 1st, 2nd, 4 and 8th weeks, while the mRNA and protein expression of NeuN, $\beta$-III-Tub and GFAP increased with time. In addition, after treatment, the MCAO rats showed decreased NSS and mRNA and protein expression of nestin, but elevated mRNA and protein expression of NeuN, $\beta$-III-Tub and GFAP at the 2nd, 4 and 8th weeks, and decreased positive expression of HNA and nestin with enhanced expression of
\end{abstract}

Correspondence to: Dr Ming Deng, Department of Orthopedics, Renmin Hospital of Wuhan University, 238 Jiefang Road, Wuchang, Wuhan, Hubei 430060, P.R. China

E-mail: dengming1983@whu.edu.cn

*Contributed equally

Key words: human bone marrow-derived mesenchymal stromal cells, neuron regeneration, transplantation, middle cerebral artery occlusion, neuronal function
NeuN, $\beta$-III-Tub and GFAP. Therefore, the present findings demonstrated that hBMSC transplantation triggered the formation of nerve cells and enhanced neuronal function in a rat model of MCAO.

\section{Introduction}

Cerebral stroke is a leading cause of mortality and long-term disability among adults in developed countries; the majority $(85 \%)$ are ischemic in nature and are caused by the closure of a major cerebral artery through an embolism or thrombus, which brings about subsequent tissue death and blood flow loss in the affected region (1). The cellular and molecular mechanisms that are responsible for the neurodegeneration induced by stroke involve acidosis, excitotoxicity, oxidative stress, bioenergetic failure and inflammation, leading to necrotic or apoptotic cell death (2). The neural network re-establishment of newborn neurons is regarded as the fundamental anatomic basis for the process of functional repair in injured brains, and many studies have been devoted to fully understanding the therapeutic mechanisms of rehabilitative treatment for patients with stroke $(3,4)$. Advances in stem cell studies over the past 20 years have led to the potential use of stem cells in the treatment of degenerative diseases and neuronal injury (5-7). It has been reported that the nerve mobilization technique can improve nerve conduction and the axonal transport system and reduce intraneural compression within the nerves to enhance circulation to the nerves (8). Importantly, one study demonstrated that preconditioned transplanted bone marrow-derived mesenchymal stromal cells (BMSCs) could initiate the de novo regeneration of gastric neuronal cells and structures that, in turn, could restore gastric contractility; this result suggested that preconditioned allogeneic BMSCs exhibit therapeutic value for the treatment of enteric nerve disorders (9).

MSCs can be applied to hematopoietic stem cell transplantation, organ transplantation, and bone and cartilage tissue repair, along with treatment for myocardial infarction and liver injury $(10,11)$. All the aforementioned functions are 
associated with the important characteristics of regeneration, but in different ways for different diseases. However, all these effects of MSCs are attributed to the regenerative power of stem cells in the lesion. The repair is believed to be induced through growth-stimulating factors provided by stem cells for tissue regeneration in the lesion (12). The supportive role of stem cells for various highly differentiated cells is responsible for these specific functions and lays the foundation for repair and regeneration in the lesion, as well as for replantation of suitable tissue $(13,14)$. It has been elucidated that the recruitment of MSCs to the injury site is accomplished through chemotaxis, including via growth factors, which are of great significance to tissue regeneration from the perspective of cell survival, proliferation, migration and differentiation (15). Experts have also reported that the pro-angiogenic properties of human umbilical cord-derived MSCs in tissue growth and regeneration are related to structural support for capillary-like structures (16).

Adult BMSCs, namely multipotent progenitors, are capable of adipogenic, osteogenic and chondrogenic differentiation and can display transdifferentiation potential beyond the restrictions of the mesenchymal lineages, such as differentiation into neurons (17-20). Due to their high plasticity and active expansion capacity, as well as their typically low immunogenicity, BMSC therapy remains a unique and attractive strategy $(21,22)$. A previous study demonstrated that following transplantation, BMSCs could develop into glial-type cells capable of improving peripheral nerve regeneration (23). Multiple animal models have been gradually developed as models for ischemic stroke, among which the middle cerebral artery occlusion (MCAO) model has been commonly adopted, especially in rodents (24). A recent study reported that MCAO stroke could cause the degeneration of nigral dopaminergic neurons in human brains, followed by ischemic neuronal death in the cerebral cortex, striatum and ischemic core (25). Studies have demonstrated that BMSC transplantation is able to enhance functional recovery after cerebral ischemia $(26,27)$. Thus, the present study was conducted to explore the mechanisms underlying the effect of hBMSC transplantation on neuron regeneration in a rat model of MCAO. It was hypothesized that hBMSC transplantation could trigger the formation of nerve cells and enhance neuronal function in a rat model of MCAO.

\section{Materials and methods}

Study subjects. A total of 70 male Sprague-Dawley (SD) rats (10 weeks old; 250-280 g) were obtained from Hunan SJA Laboratory Animal Co., Ltd. All treatments on animals were in accordance with 'On the Guidance to Take Care of the Experimental Animals' issued by the Ministry of Science and Technology of China. Adult marrow was obtained from two surgical patients (female; 24 and 28 years old) and three orthopedic trauma patients ( 1 female, 34 years old; 2 males, 26 and 38 years old) in Renmin hospital of Wuhan University between September 2018 and December 2018. According to 'Managerial Regulation of the Medical Institutions Administrative Rules', promulgated by the State Council of China, the experimental procedures and risks were announced to the subjects, and informed consent was received from all subjects. All experimental animal operating procedures were in line with the US National Institutes of Health laboratory animal care and usage guidelines.

Isolation and culture of hBMSCs. In a sterile environment, $20 \mathrm{ml}$ of healthy human marrow was extracted for the isolation of leukomonocytes, which were then used to obtain mononuclear cells through the density-gradient method (28). Next, the mononuclear cells were inoculated in DMEM/F12 (cat. no. 51985042; Gibco; Thermo Fisher Scientific, Inc.) containing $10 \% \mathrm{FBS}$ and $1 \%$ glutamine at a concentration of $1 \times 10^{9} \mathrm{I}^{-1}$, and cultured in a $5 \% \mathrm{CO}_{2}$ incubator with saturated humidity at $37^{\circ} \mathrm{C}$. The solution was changed every 3 days. When the cell confluence reached $80 \%$, the cells were detached using a mixed solution of $2.5 \mathrm{~g} / 1$ trypsin and $0.2 \mathrm{~g} / 1$ EDTA, and subcultured at a ratio of 1:2 for amplification.

Identification of hBMSCs. Cells at passage 5 were inoculated in a 24-well plate with a prepared sterile coverslip at a density of $4 \times 10^{4} / \mathrm{ml}$ and cultured at $37^{\circ} \mathrm{C}$ under $5 \% \mathrm{CO}_{2}$. The cells were fixed with $4 \%$ paraformaldehyde at room temperature for $30 \mathrm{~min}$, treated with $0.3 \%$ TritonX-100 at room temperature for $30 \mathrm{~min}$, and blocked with goat serum (Thermo Fisher Scientific Inc.) for $1 \mathrm{~h}$ at room temperature. Next, the cells were incubated at room temperature for $3 \mathrm{~h}$ with the following primary antibodies: Rat anti-human CD34 (bsm-16538M, 1:100; BIOSS), CD44 (bsm-51065M, 1:100; BIOSS) and CD45 (bs-4819R, 1:100; BIOSS). After three washes with PBS with Tween-20 (PBST), the cells were incubated with FITC-labeled secondary goat anti-mouse antibody (bs-0293P, 1:100, BIOSS) at room temperature for $1 \mathrm{~h}$, avoiding exposure to light. Finally, the cells were rinsed three times with PBST, mounted with neutral resin, and observed and photographed under a confocal laser scanning microscope (LSM 700; Carl Zeiss $\mathrm{AG})$; the number of positive cells were counted in a random field per view.

MCAO model establishment and grouping. According to the modified Longa method (29), the rat model of MCAO was established. At $2 \mathrm{~mm}$ behind and $5 \mathrm{~mm}$ to the right of the fontanelle, laser Doppler flowmetry was used to monitor the blood flow in the brain before occlusion, after occlusion and after reperfusion. The ischemia was successfully induced when the blood flow after occlusion decreased to $<30 \%$ of that before occlusion, and the reperfusion was shown to be successful when the blood flow increased to $70 \%$ of that before occlusion with no occurrence of basilar hemorrhage (29). During the surgery, an animal pulse oximeter was used to monitor the arterial oxygen saturation, breathing rate and heart rate of all rats. After the surgery, water and food were offered to the rats. According to the random number table method, the established rat models were randomly divided into treatment (injected with hBMSCs; $n=35$ ) and control (injected with PBS; $n=35$ ) groups. All rats received $8.0 \mathrm{~Gy} \gamma$-radiation in the whole body to suppress immunity, and the transplantation was conducted on the 3rd day after radiotherapy. Rats in the treatment group were stereotactically injected with $0.5 \mathrm{ml}$ of hBMSCs $\left(1 \times 10^{7}\right.$ cells/rat) through the cerebral cortex, and rats in the control group were stereotactically injected with the same amount of PBS through the cerebral cortex. The Neurological Severity Score (NSS) was determined at 1, 2, 4 and 8 weeks 
after transplantation. Of note, 5 and 8 rats succumbed in the treatment and control groups, respectively. Thus, 6 surviving rats in each group were anesthetized and sacrificed by cervical vertebra dislocation to obtain whole brain tissues; of these tissues, one part was used for reverse transcription-quantitative PCR (RT-qPCR) and the other part was used for western blot analysis. The brain tissues at 1 week after transplantation were used for triphenyl-2,3,5-tetrazoliumchloride (TTC) staining, and the brain tissues at 8 weeks after transplantation were used for immunohistochemistry.

Stereotaxic injection. Rats were placed in the middle of the stereotaxic apparatus. The front teeth of the rats were locked on the anterior clamp. The clamp was lowered and tightened. The ear bars were inserted into the ear canal to stabilize the head; the insertion distance was limited to avoid any damage to the inner ear. The surgical site was sterilized by $70 \%$ ethanol and $10 \%$ iodophor using a cotton swab (two times). An incision was created at $1.5 \mathrm{~cm}$ above the ears (toward the nose) and extended to $0.5 \mathrm{~cm}$ below the ears. The incision was further extended vertically to the middle of the head to expose the bregma, followed by slow removal of the membrane on the surface of the skull using a sterilized cotton swab. The syringe $(1 \mu \mathrm{l})$ was filled with cells with no bubbles and then placed in the intersection of the bregma and interaural line where the point was set at zero, with the coordinates determined using brain atlases. Then, the syringe was slightly raised to place the drill directly above the target coordinate at a $\sim 45^{\circ}$ angle to the skull. The reduction of resistance signaled the initiation of the next step. It is necessary that drill not be put into the brain in order to prevent cortical damage. The syringe was put at a depth of $<2 \mathrm{~mm}$ and the injection started at a speed of $0.2 \mu \mathrm{l} / \mathrm{min}$. It is necessary to ensure that the syringe does not slip lower than the desired depth. If this occurs, the syringe should be gently lifted back to the desired depth. Then, 2 min after the injection was completed, any residual cells were absorbed. The syringe was slowly lifted for injection into the other hemisphere. The scalp was sutured and sterilized with iodophor (30).

NSS. The NSS system was used for the evaluation of functional nerve damage and recovery in rats in the treatment and control groups at 1,2, 4 and 8 weeks after surgery (31). The scores were as follows: $13-18$ points was regarded as severe neurological impairment; 7-12 points as moderate nerve function damage; and 1-6 points as light nerve function damage. The NSS evaluates the motor function, beam test, sensory function, reflex activity and other responses that can comprehensively reflect nerve function. Higher scores indicate more severe symptoms, and lower scores indicate lighter symptoms (Table I).

TTC staining. At 1 week after transplantation, the rats were anesthetized with sodium pentobarbital (50 mg/kg; i.p.), and the rat brains were removed carefully. Next, the brains were cut into $1-\mathrm{mm}$ coronal sections using a vibratome. The brain sections were immersed in $2 \%$ TTC solution with normal saline at $37^{\circ} \mathrm{C}$ for $30 \mathrm{~min}(32)$.

$R T-q P C R$. The whole brain tissue samples were removed at 1,2 , 4 and 8 weeks after transplantation and NSS determination. The total RNA was extracted using TRIzol ${ }^{\mathrm{TM}}$ (cat. no. 15596-018;
Invitrogen; Thermo Fisher Scientific, Inc.), and the absorbance (A)260/A280 and RNA concentration were detected using a NanoDrop ultraviolet spectrophotometer (NanoDrop 2000; Thermo Fisher Scientific, Inc.). Five $\mu \mathrm{g}$ of cDNA was reverse transcribed according to the manufacturer's instructions of the cDNA kit (cat. no. K1622; Fermentas Inc., Ontario, CA, USA). The cDNA was synthesized via RT, according to the instructions of the Applied Biosystems StepOne ${ }^{\mathrm{TM}}$ (cat. no. 4376357, Thermo Fisher Scientific, Inc.) and StepOnePlus ${ }^{\mathrm{TM}}$ Real-Time PCR Systems (cat. no. 4376600, Thermo Fisher Scientific, Inc., Waltham, MA, USA) at room temperature. The PCR (cat. no. A25780, SYBR Green I, Thermo Fisher Scientific, Inc.) was conducted using the ABI 7500 RT-qPCR instrument (cat. no. AM1005; Invitrogen; Thermo Fisher Scientific, Inc.). The reaction conditions for nestin, $\beta$-III-tubulin ( $\beta$-III-Tub), glial fibrillary acidic protein (GFAP) and neuronal nuclear antigen $(\mathrm{NeuN})$ were as follows: Pre-denaturation at $95^{\circ} \mathrm{C}$ for $3 \mathrm{~min}$, a total of 38 cycles of denaturation $95^{\circ} \mathrm{C}$ for $30 \mathrm{sec}$, annealing at $60^{\circ} \mathrm{C}$ for $40 \mathrm{sec}$ and extension at $60^{\circ} \mathrm{C}$ for $40 \mathrm{sec}$. The fold changes between the experimental group and the control group were calculated by means of the $2^{-\Delta \Delta \mathrm{Cq}}$ method for relative quantification. Each sample had three duplicates and all primers used above were synthesized by Sangon Biotech Co., Ltd. (Table II).

Western blot analysis. The whole brain tissue samples were removed 1, 2, 4 and 8 weeks after transplantation and NSS. Next, $80 \mathrm{mg} / \mathrm{kg}$ samples were added to $1 \mathrm{ml}$ of tissue lysate (cat. no. 89900, Thermo Fisher Scientific, Inc), $40 \mathrm{mg}\left(\sim 5 \times 10^{6}\right.$ cells) of wet cell precipitation, ground into homogenate in an ice bath, and lysed to obtain protein lysate at $4^{\circ} \mathrm{C}$ for $30 \mathrm{~min}$ with shaking every $10 \mathrm{~min}$. Next, the samples were centrifuged at $25,764 \mathrm{x} \mathrm{g}$ at $4^{\circ} \mathrm{C}$ for $20 \mathrm{~min}$ to eliminate the lipid layer. The liquid supernatant was obtained, and a bicinchoninic acid kit (cat. no. 20201ES76; Yeasen, Inc.) was adopted to detect the protein concentration of each sample. Deionized water was used to adjust the loading sample to $30 \mu \mathrm{g}$ protein/lane. Next, the proteins were separated by $10 \%$ SDS-PAGE, transferred onto a nitrocellulose membrane and blocked with 5\% skimmed milk powder at $4{ }^{\circ} \mathrm{C}$ overnight. Next, the membrane was incubated with rabbit anti- $\beta$-III-Tub (cat. no. ab18207; $1: 1,000$ ), GFAP (cat. no. ab7260; 1:5,000), nestin (cat. no. ab221660; $1: 1,000)$ and NeuN (cat. no. ab177487; 1:2,000) in a sealed plastic bag at $4^{\circ} \mathrm{C}$ overnight, followed by three PBST washes (5 min each) at room temperature. Next, the membrane was incubated with horseradish peroxidase (HRP)-labeled goat anti-rabbit secondary antibody immunoglobulin G (IgG; cat. no. ab6721; $1: 5,000)$ at $37^{\circ} \mathrm{C}$ for $1 \mathrm{~h}$ and washed three times with PBST (5 min each) at room temperature. The membrane was soaked in ECL solution (cat. no. ECL808-25; Biomiga, Inc.), subjected to the reaction at the room temperature for 1 min with the liquid eliminated, and then developed in the dark. GAPDH (1:10,000, ab181602, Abcam) was used as the internal reference, and the relative protein expression was calculated via the ratio of the gray value of the target band to that of the internal reference band (Quantity One, v4.6.6, Bio-Rad Laboratories, Inc.).

Immunohistochemistry. A total of 8 weeks after transplantation, the rats were anesthetized and subjected to reperfusion with normal saline and $40 \mathrm{~g} / \mathrm{l}$ paraformaldehyde through the heart. 
Table I. Detailed standards for neurological severity score points.

\begin{tabular}{|c|c|c|}
\hline Items & Contents & Points \\
\hline \multirow[t]{3}{*}{ Electric shock experiments } & Flexion of forelimb & 1 \\
\hline & Flexion of hindlimb & 1 \\
\hline & Head moved $>10^{\circ}$ to vertical axis within $30 \mathrm{sec}$ & 1 \\
\hline \multirow[t]{4}{*}{ Motor tests } & Normal walk & 0 \\
\hline & Inability to walk straight & 1 \\
\hline & Circling toward paretic side & 2 \\
\hline & Falling down to paretic side & 3 \\
\hline \multirow[t]{2}{*}{ Sensory tests } & Placing test (visual and tactile test) & 1 \\
\hline & $\begin{array}{l}\text { Proprioceptive test (deep sensation, pushing paw against table edge to } \\
\text { stimulate limb } 1 \text { muscles) }\end{array}$ & 1 \\
\hline \multirow[t]{7}{*}{ Beam balance tests } & Steady posture & 0 \\
\hline & Grasps side of beam & 1 \\
\hline & Hugging beam and 1 limb falls down from beam & 2 \\
\hline & Hugging beam and 2 limbs fall down from beam, or spins on beam $(60 \mathrm{sec})$ & 3 \\
\hline & Trying to balance on beam but falling off ( $40 \mathrm{sec})$ & 4 \\
\hline & Trying to balance on beam but falling off $(20 \mathrm{sec})$ & 5 \\
\hline & Falling off without attempt to balance or hang on to beam $(20 \mathrm{sec})$ & 6 \\
\hline \multirow{4}{*}{$\begin{array}{l}\text { Reflex absence and } \\
\text { abnormal movements }\end{array}$} & Pinna reflex (head shaking when auditory meatus is touched) & 1 \\
\hline & Corneal reflex (eyes blinking when cornea is lightly touched with cotton) & 1 \\
\hline & Startle reflex (motor response to a brief noise from snapping a clipboard paper) & 1 \\
\hline & Seizures, myoclonus, myodystony & 1 \\
\hline Maximum points & & 18 \\
\hline
\end{tabular}

Table II. Primer sequences for the reverse transcriptionquantitative PCR.

\begin{tabular}{ll}
\hline Gene & \multicolumn{1}{c}{ Primer sequence (5'-3') } \\
\hline Nestin & F: CAGCTGGCGCACCTCAAGATG \\
& R: AGGGAAGTTGGGCTCAGGACTGG \\
F-III-Tub & F: AGATGTACGAAGACGACGAGGAG \\
& R: GTATCCCCGAAAATATAAACACAAA \\
GFAP & F: GTGGGCAGGTGGGAGCTTGATTCT \\
& R: CTGGGGCGG CCTGGTATGACA \\
NeuN & F: GGGTTTGGGTTTGTACTTTGAA \\
& R: AGACTGCTCCTACCACAGGGTTTAG \\
GAPDH & F: TCCCCACTGCCAACGTGTCAGTG \\
& R: ACCCTGTTGCTGTAGCCAAATTCG
\end{tabular}

$\beta$-III-Tub, $\beta$-III-tubulin; GFAP, glial fibrillary acidic protein; NeuN, neuronal nuclear antigen; $\mathrm{R}$, reverse, $\mathrm{F}$, forward.

Cervical vertebra dislocation was performed to obtain the brain tissues. The brain tissues were placed in normal saline at $1-4^{\circ} \mathrm{C}$, embedded in optimal cutting temperature compound and cut into 5- $\mu \mathrm{m}$ frozen sections. Next, the sections were treated with $3 \% \mathrm{H}_{2} \mathrm{O}_{2}$ for $30 \mathrm{~min}$ to block endogenous peroxidase activity, incubated in $0.3 \%$ Triton X-100/0.1\% BSA (Thermo Fisher Scientific, Inc.) at $37^{\circ} \mathrm{C}$ for $2 \mathrm{~h}$, and incubated in a wet box at $4^{\circ} \mathrm{C}$ overnight with rabbit anti $\beta$-III-Tub polyclonal antibody (cat. no. ab18207; 1:200), GFAP (cat. no. ab7260; 1:100), nestin (cat. no. ab92391; 1:250), NeuN (cat. no. ab104224; 1:5,000), HNA (cat. no. ab215396; 1:100) and GAPDH (cat. no. ab8245; $1: 1,000)$. PBS at a concentration of $0.01 \mathrm{~mol} / 1$ was adopted as the NC. Following the incubation, the sections were washed three times with PBST, incubated with HRP-labeled rabbit anti-mouse secondary antibody IgG (cat. no. ab6725; 1:1,000) or goat anti-rabbit secondary antibody $\operatorname{IgG}$ (cat. no. ab6721; $1: 1,000)$ at $37^{\circ} \mathrm{C}$ for $1 \mathrm{~h}$, and washed three times with PBST. Lastly, the sections were stained with diaminobenzidine at room temperature for $10 \mathrm{~min}$, and brown staining was verified as a positive reaction. A total of five visual fields were randomly selected in each section for photographing under an inverted microscope (XDS-800D; magnification, x200; Canon, Inc.); the positive expression rate was calculated.

Statistical analysis. SPSS 21.0 (IBM Corp.) was used for the data analysis. The measurement data are presented as the mean \pm standard deviation. The experiment was repeated 3 times. An independent sample t-test was used for comparisons between two groups. The enumeration data are shown as the typical value of the cases. The $\chi^{2}$ test was applied for comparisons between two groups. $\mathrm{P}<0.05$ was considered to indicate a statistically significant difference.

\section{Results}

Identification of hBMSCs. The immunofluorescence assay results showed that no positive expression of CD34 and 
A

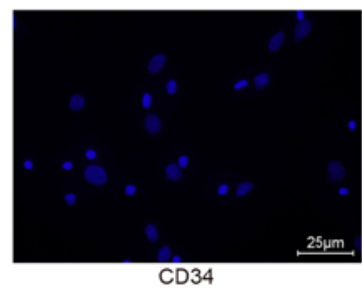

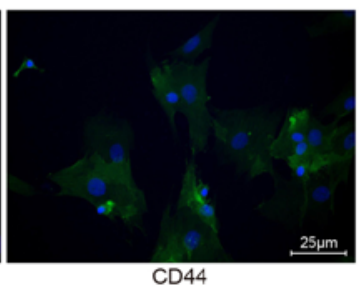

CD44

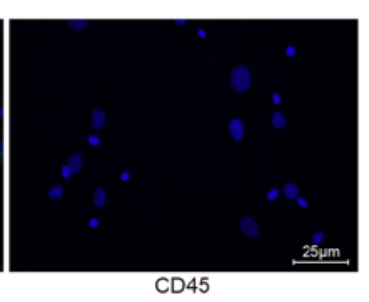

CD45

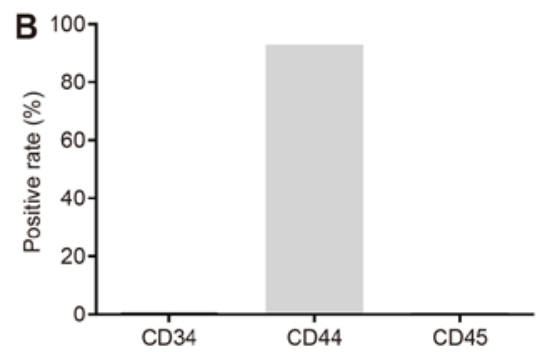

Figure 1. Expression of BMSC surface markers (CD34, CD44 and CD45) detected by the immunofluorescence assay and the positive rate of CD44 is $92.75 \%$, indicating that most of the cells we isolated are human bone marrow mesenchymal stem cells, conforming to further experimental requirements. (A) CD34, CD44 and CD45, as examined by immunofluorescence assay (magnification, $x 400$ ). Green, surface markers; blue, nuclei. (B) Positive expression rates of CD34, CD44 and CD45.

CD45 appeared in the hBMSCs after isolation and culture, suggesting that the isolated cells were not hemopoietic stem cells, since both the surface molecules CD34 and CD45 should be positive if so. The positive expression rate of CD44 was $92.75 \%$, indicating that the majority of the isolated cells were hBMSCs, due to the fact that CD44 serves as the main surface marker for cell identity concerning hBMSCs (33). These findings demonstrated that the cultured hBMSCs in the present study were suitable for use in further experiments (Fig. 1).

Successful establishment of the MCAO rat model. For all 70 rats in the treatment and control groups, after ligation of the middle cerebral artery by strings with diameters of 0.36 and $0.38 \mathrm{~mm}$, the cerebral blood flow of the 70 mice was reduced to $<30 \%$ of that before occlusion and, after reperfusion, increased to $>70 \%$ of that before occlusion. The following symptoms occurred $24 \mathrm{~h}$ after anesthesia: Lack of contraction of the left limb upon pain stimulation, occurrence of circling or dumping and the inability to extend straight forward upon lifting the tail. This observation indicated that the model establishment rate was $100 \%$. In total, five rats succumbed in the treatment group, with a survival rate of $85.7 \%$, and eight rats succumbed in the control group, with a survival rate of $77.1 \%$, three died in the first week, six died in the second week, and four died in the third week.

NSS decreases remarkably in MCAO rats after $h B M S C$ transplantation. NSS was applied to evaluate the nerve function damage and recovery condition of rats in the treatment and control groups at 1, 2, 4 and 8 weeks after surgery. Scores of 13-18 points were regarded as severe neurological impairment, 7-12 points as moderate nerve function damage, and 1-6 points as light nerve function damage. The NSS evaluates motor function, the beam test, sensory function, reflex activity and other indicators, which comprehensively reflect nerve function. The higher the score, the more serious the symptoms. The results of NSS evaluation revealed that the rats mainly showed moderate nerve function damage 1 week after surgery, and this damage decreased with time. The NSS presented a generally decreasing trend both in the treatment and control groups 1,2, 4 and 8 weeks after surgery. Compared with the control group, the NSS decreased significantly in the treatment group 2, 4 and 8 weeks after surgery ( $\mathrm{P}<0.05$; Fig. 2).

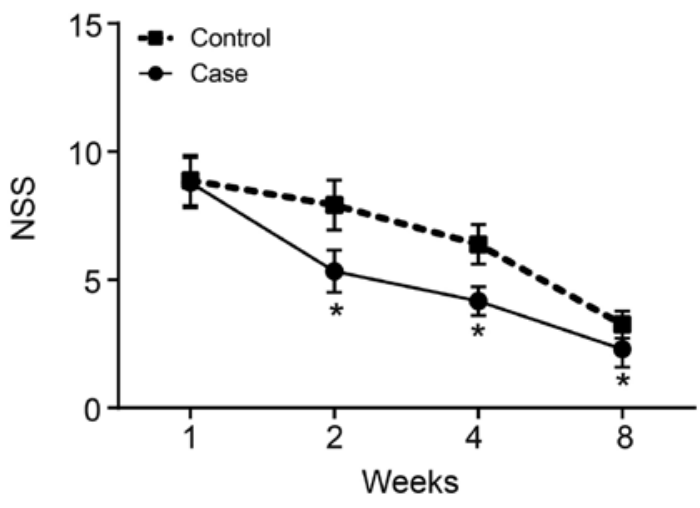

Figure 2. NSS significantly decreases in MCAO rats after hBMSC transplantation. ${ }^{*} \mathrm{P}<0.05$ vs. control group. NSS, neurological severity score; hBMSCs, human bone-marrow-derived mesenchymal stem cells; MCAO, middle cerebral artery occlusion.

Infarct areas are reduced in MCAO rats after hBMSC transplantation. TTC staining was conducted using the brain tissues of MCAO rats to observe the severity of obstruction in the rats according to the infarct areas. The results of the TTC staining revealed that rats in the control group showed larger infarct areas than those in the treatment group $(\mathrm{P}<0.05$; Fig. 3). Therefore, MCAO rats showed reduced infarct areas after hBMSC transplantation.

mRNA expression of neuronal biomarkers increases after hBMSC transplantation in MCAO rats. RT-qPCR was conducted to examine the mRNA expression of brain tissue neuronal biomarkers. The results showed that the mRNA expression of nestin decreased in the control and treatment groups with time at 1, 2, 4 and 8 weeks after hBMSC transplantation, and it was lower in the treatment group than in the control group $(\mathrm{P}<0.05$; Fig. $4 \mathrm{~A})$. The mRNA expression of NeuN, $\beta$-III-Tub and GFAP was elevated significantly in both groups and was higher in the treatment group than in the control group (all $\mathrm{P}<0.05$; Fig. 4B-D).

Protein expression of nestin, NeuN, $\beta$-III-Tub and GFAP increases after hBMSC transplantation. Western blot analysis was applied to examine the protein expression of nestin, NeuN, $\beta$-III-Tub and GFAP 1, 2, 4 and 8 weeks after hBMSC transplantation, and the results showed that the protein expression of nestin in both groups decreased with time and 
A

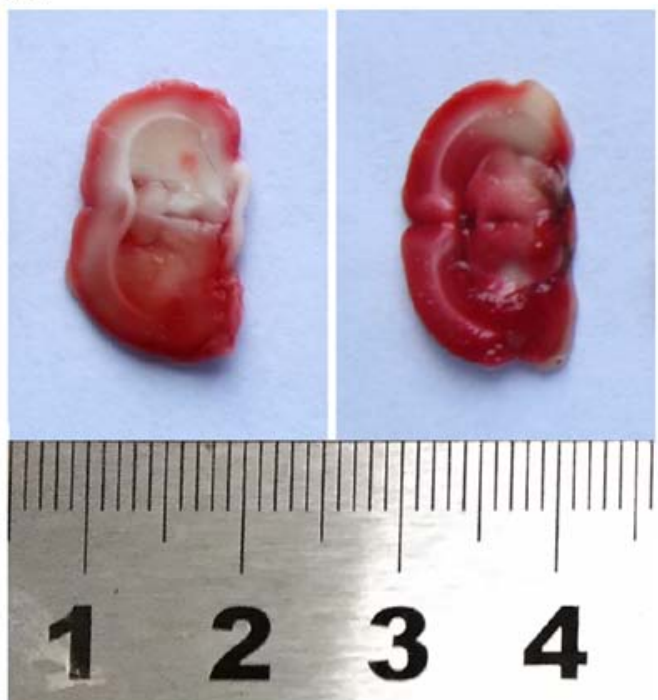

Control
B

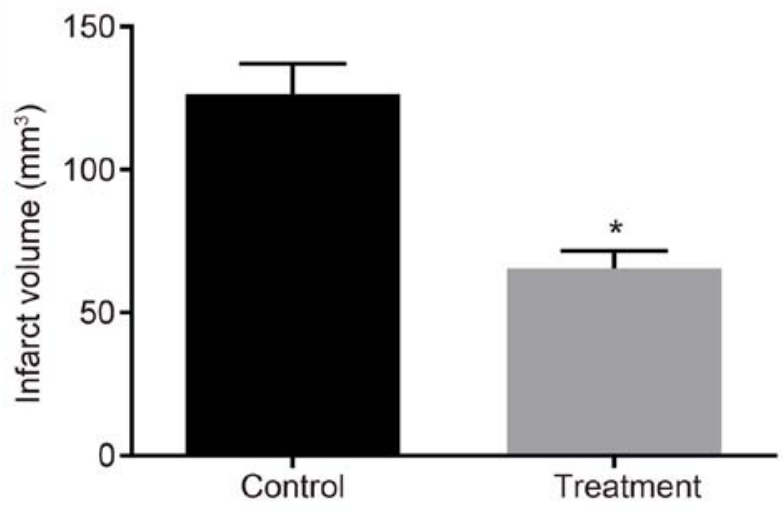

Figure 3. Decreased infarct areas are found in MCAO rats after hBMSC transplantation. (A) The brain tissues of rats in the control and treatment groups, as examined by triphenyl-2,3,5-tetrazoliumchloride staining. (B) The infarct volumes of rats in the control and treatment groups. "P<0.05 vs. control group. MCAO, middle cerebral artery occlusion; hBMSCs, human bone marrow-derived mesenchymal stem cells.
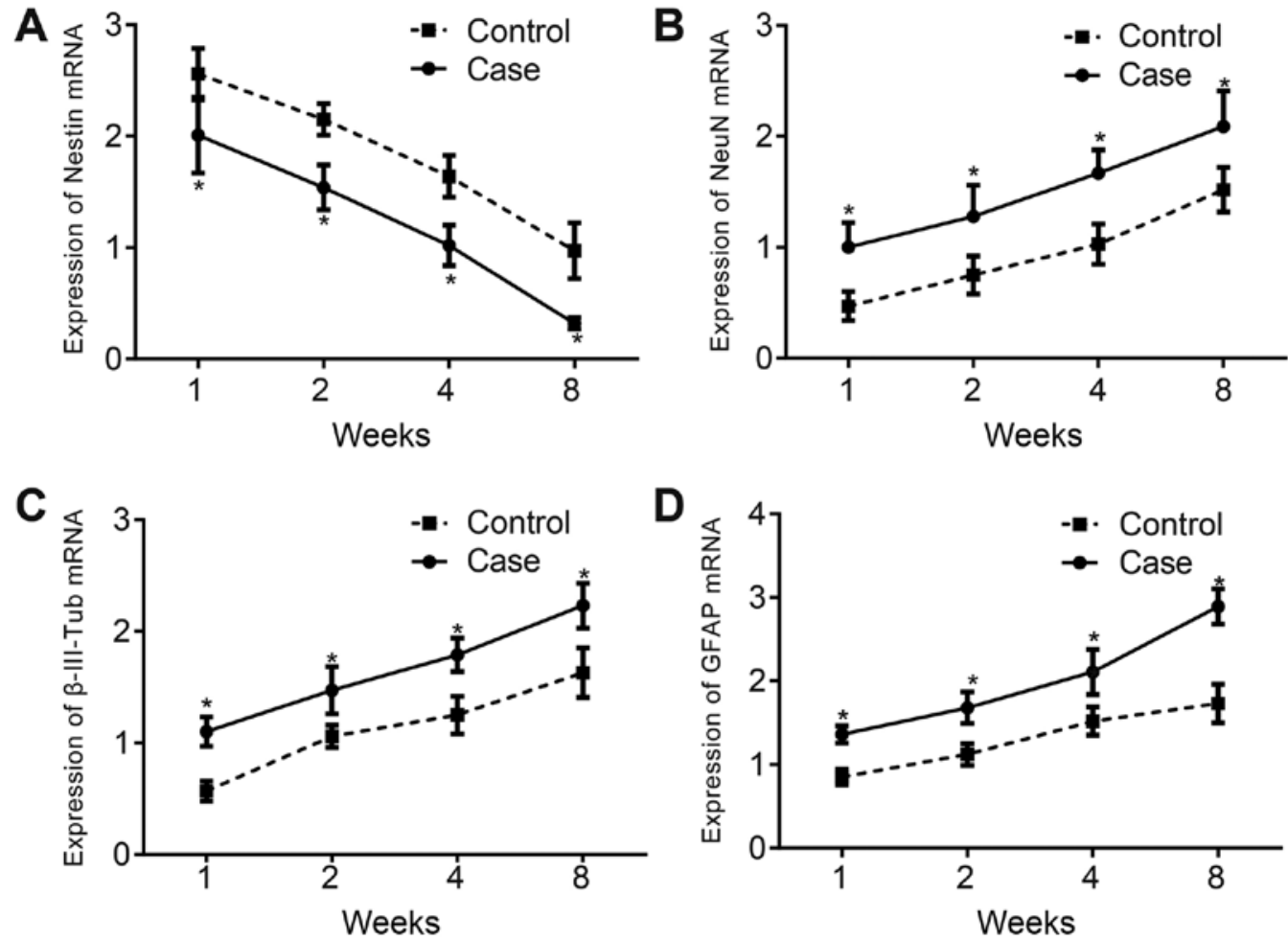

Figure 4. mRNA expression of nestin, NeuN, $\beta$-III-Tub and GFAP increases after hBMSC transplantation. (A) mRNA expression of nestin at $1,2,4$ and 8 weeks after hBMSC transplantation. (B) mRNA expression of NeuN at 1,2,4 and 8 weeks after hBMSC transplantation. (C) mRNA expression of $\beta$-III-Tub at 1,2, 4 and 8 weeks after hBMSC transplantation. (D) mRNA expression of GFAP at 1, 2, 4 and 8 weeks after hBMSC transplantation. "P<0.05 vs. respective control group. $\beta$-III-Tub, $\beta$-III-tubulin; GFAP, glial fibrillary acidic protein; NeuN, neuronal nuclear antigen; hBMSCs, human bone marrow-derived mesenchymal stem cells.

was significantly decreased in the treatment group compared with the control group $(\mathrm{P}<0.05)$. The protein expression of NeuN, $\beta$-III-Tub and GFAP was elevated in both groups, and increased significantly in the treatment group compared with the control group $(\mathrm{P}<0.05$; Fig. 5).
Positive expression rates of NeuN, $\beta$-III-Tub and GFAP are increased after hBMSC transplantation. At 8 weeks after hBMSC transplantation, immunohistochemical staining showed that the positive expression rates of HNA, nestin, NeuN, $\beta$-III-Tub and GFAP after hBMSC transplantation in 

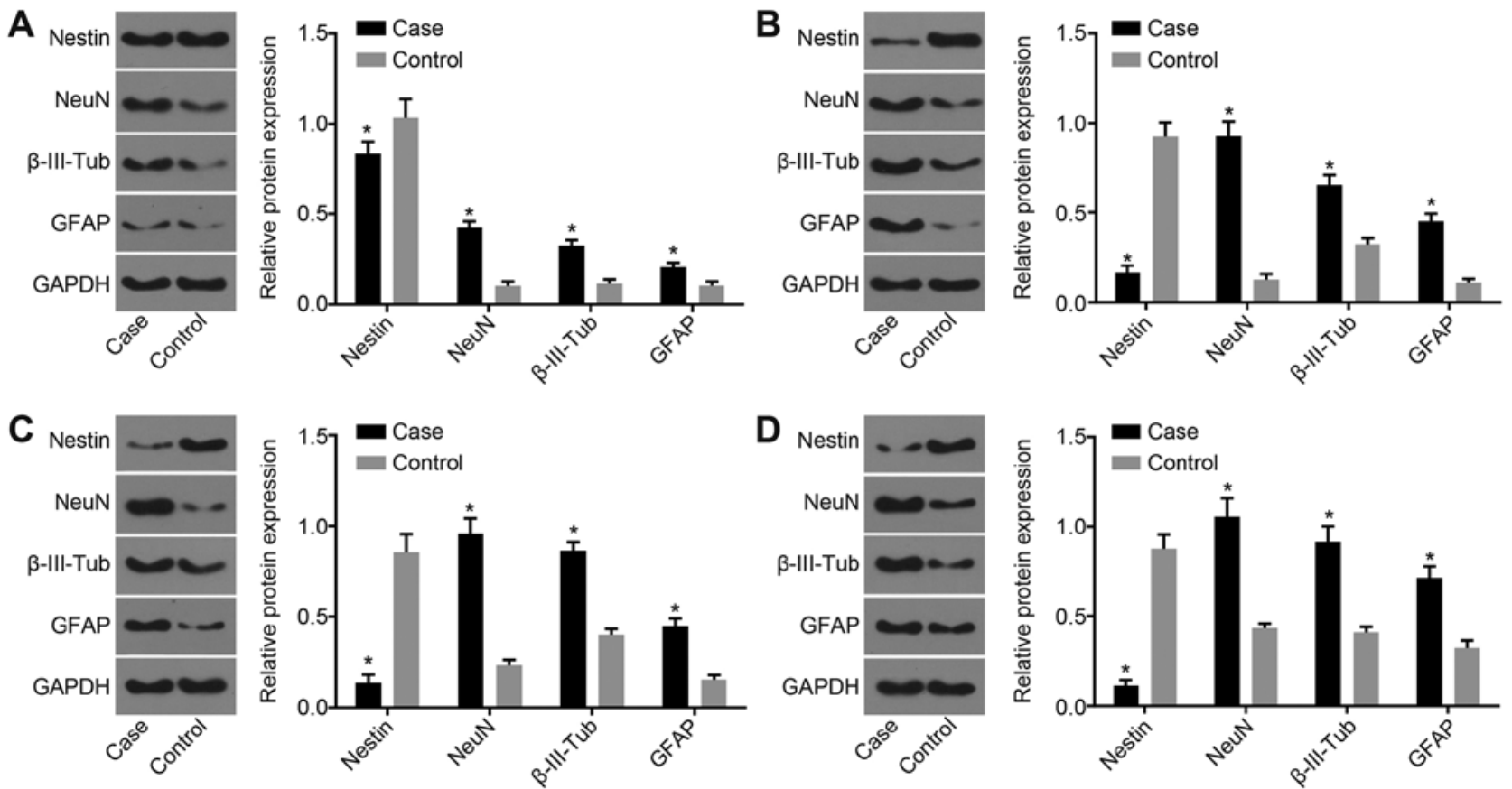

Figure 5. Protein expression of nestin, NeuN, $\beta$-III-Tub and GFAP increases after hBMSC transplantation. (A) Protein bands for nestin, NeuN, $\beta$-III-Tub, GFAP and GAPDH 1 week after hBMSC transplantation. (B) Protein bands for nestin, NeuN, $\beta$-III-Tub, GFAP and GAPDH 2 weeks after hBMSC transplantation. (C) Protein bands for nestin, NeuN, $\beta$-III-Tub, GFAP and GAPDH 4 weeks after hBMSC transplantation. (D) Protein bands for nestin, NeuN, $\beta$-III-Tub, GFAP and GAPDH 8 weeks after hBMSC transplantation. ${ }^{*} \mathrm{P}<0.05$ vs. respective control group. $\beta$-III-Tub, $\beta$-III-tubulin; GFAP, glial fibrillary acidic protein; NeuN, neuronal nuclear antigen; hBMSCs, human bone marrow-derived mesenchymal stem cells.

the treatment group were $14.8,11.8,78.0,74.2$ and $36.2 \%$, respectively, and those in the control group were 72.2, 65.8, $22.2,20.2$ and $9.4 \%$, respectively. These results suggested that the positive expression rates of HNA and nestin decreased significantly, while those of NeuN, $\beta$-III-Tub and GFAP after hBMSC transplantation in the treatment group were elevated significantly compared with those in the control group (all $\mathrm{P}<0.05$; Fig. 6).

\section{Discussion}

Stroke is a major cause of mortality worldwide, and it remains a significant cause of long-term disability among adults; $87 \%$ of stroke cases are ischemic in origin and occur in the territory of the middle cerebral artery (34). It has been demonstrated that BMSC transplantation can improve functional recovery after cerebral ischemia $(35,36)$. It has been reported that the nerve mobilization technique can improve nerve conduction and the axonal transport system, and reduce intraneural compression within the nerves, thus improving circulation to the nerves (8). Therefore, the present study aimed to explore the mechanisms underlying the effect of hBMSC transplantation on neuron regeneration in a rat model of MCAO, to develop a therapeutic method for enhancing nerve function following stroke. Compared with the control group, the case group exhibited significantly decreased NSS in the 2nd, 4 and 8th weeks compared with the control group. The modified NSS test has been commonly adopted to evaluate neurological function (37). One study tested the hypothesis that upon transplantation into the ischemic boundary zone, hBMSCs could differentiate, survive and improve functional recovery after MCAO (38). Neurological disorders may reduce the range of motion of the knee joint, which can affect the physiological movement of the spine and the pelvis, triggering excessive pelvic inclination during walking (39). Adult neurogenesis often consists of one or more of the following processes: Survival, proliferation, migration and differentiation (40). Additionally, it is well-known that neurogenesis is closely associated with the cell cycle, and neural stem cells may play roles by exiting the cell cycle (41). In addition, modification of the molecular signals and morphogens in the microenvironment of the developing brain influences stem cell survival and differentiation (42). Neurons have been found to be generated successfully from stem cells after culture, which provides a method to develop a stem cell-based transplantation strategy for human patients (43). Transplantation of hBMSCs reduces stereotypical behaviors and cognitive rigidity, and improves social behavior (44). In the present study, the NSS decreased significantly after hBMSC transplantation, indicating that hBMSC transplantation could promote nerve cell regeneration and enhance the neuronal function of human patients, in line with the aforementioned previous studies.

Compared with the control group, in the case group, the mRNA and protein expression and positive expression rates of nestin were significantly decreased, while those for NeuN, $\beta$-III-Tub and GFAP were elevated. Nestin, a class VI intermediate filament protein, was originally regarded as a neuronal stem cell biomarker in the development of the central nervous system, and it has been widely used in this capacity (45). Nestin has often been observed in the anterior, intermediate and neural lobes among scattered cells (46). GFAP is commonly regarded as a specific predictor of neurological outcome and brain damage in patients with traumatic brain injury (47). NeuN, a nuclear protein, is commonly expressed in mature post-mitotic 


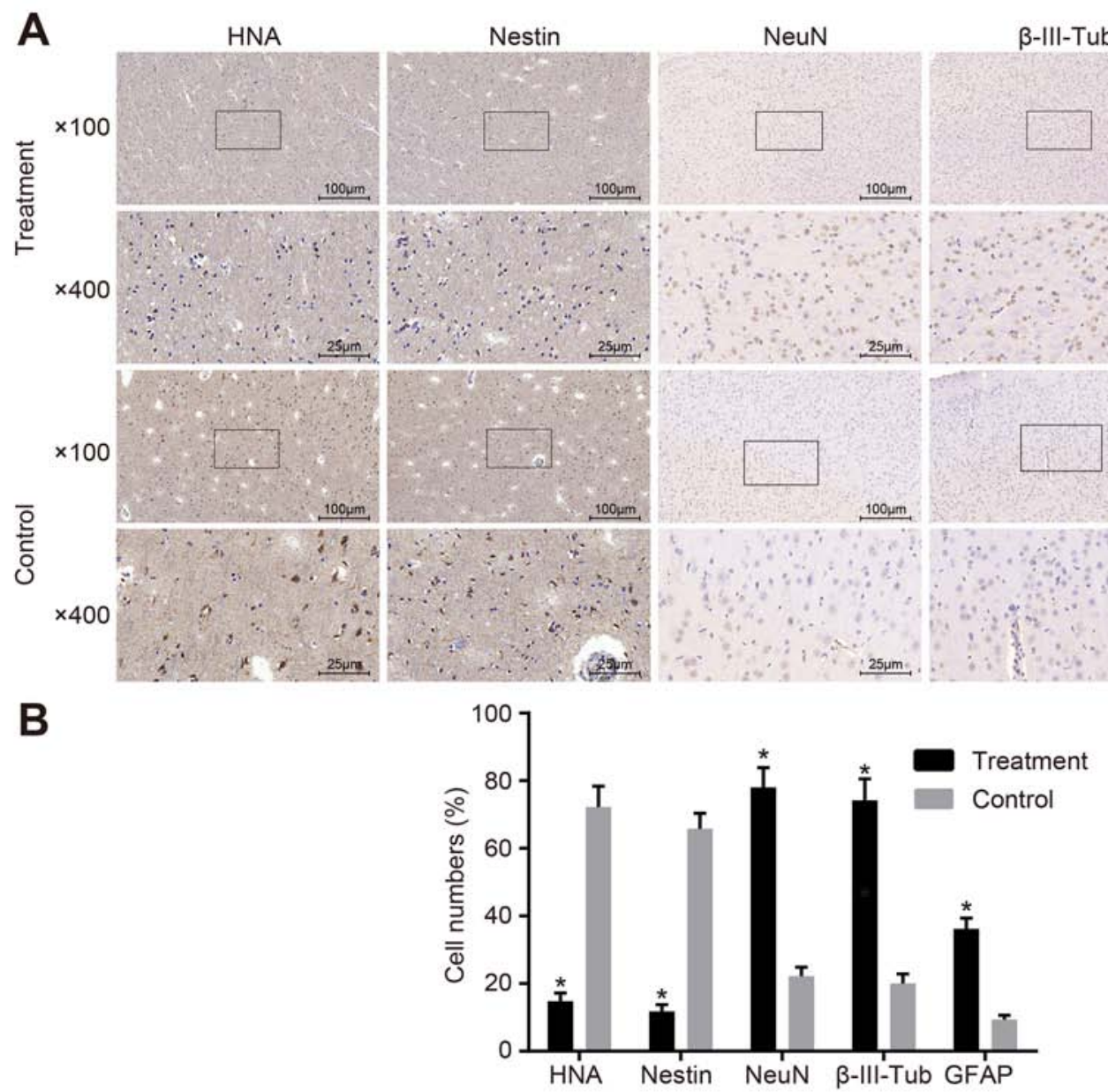

Figure 6. Differentiation of hBMSCs into neural cells in the rat brain after transplantation. (A) HNA, nestin, NeuN, $\beta$-III-Tub and GFAP in the control and treatment groups, as examined by immunohistochemistry. Cell staining after hBMSC transplantation in the case and control groups is presented. (B) Cell numbers for HNA, nestin, NeuN, $\beta$-III-Tub and GFAP in the treatment and control groups. ${ }^{*}$ P<0.05 vs. control group. $\beta$-III-Tub, $\beta$-III-tubulin; GFAP, glial fibrillary acidic protein; HNA, human neutrophil alloantigen; NeuN, neuronal nuclear antigen; hBMSCs, human bone marrow-derived mesenchymal stem cell.

neurons, and altered NeuN expression is a useful marker for immature or injured neurons (48). It is often localized in the perinuclear cytoplasm and the nuclei of neurons in the central nervous system (49). One study reported that BMSCs contain a highly mobile number of $\mathrm{C}-\mathrm{X}-\mathrm{C}$ chemokine receptor type 4-A-positive cells that express mRNA for different biomarkers of early tissue-committed stem cells, including $\beta$-III-Tub, nestin, NeuN and GFAP (50). Another study demonstrated that co-culture of amyloid- $\beta$ treated neuronal progenitor cells with BMSCs markedly increases the expression of Ki-67, GFAP and ELAV like RNA binding protein 4, indicating that BMSCs can modulate endogenous adult neurogenesis for Alzheimer's disease treatment (51). Thus, it was concluded that hBMSC transplantation may promote nerve cell regeneration and the neuronal function of human patients. All of these effects of MSCs described above are attributed to the ability of stem cells to regenerate in lesions and the determinants of growth factors and structurally supported release (52). Repair is thought to be induced by growth stimulators provided by stem cells for tissue regeneration in injury. Importantly, the regenerative effects of MSCs under injury conditions are closely associated with the release of cell protection and survival-related factors $(53,54)$. The support of stem cells for various highly differentiated cells is the cause of this specific function, laying the foundation for the repair and regeneration of lesions and the replantation of appropriate tissues.

MSCs derived from adult tissues present an potentially important progenitor cell source for applications in tissue engineering and regenerative medicine. Modalities may include direct implantation and/or ex vivo tissue engineering, in combination with biocompatible/biomimetic biomaterials and/or natural or recombinantly-derived biologics. MSCs may also be considered for gene therapy applications for the delivery of genes or gene products $(13,14)$. Another prospect for the future is the use of MSCs to create 'off-the-shelf' tissue banks. Karow et al (55) demonstrated that reprogramming of somatic cells into neurons offers a novel avenue for cell-based therapy for neurodegenerative diseases; a major challenge for this process is that it is uncertain whether the adult brain contains a population of cells that can be transformed directly into somatic cells. Crisan et al (56) reported that the vascular wall contains a number of progenitor cells that may be associated with the origin of elusive MSCs and other related adult stem cells. To fully harness the potential of these cells, future studies should be directed towards ascertaining their cellular and molecular characteristics for optimal identification, isolation and expansion, and to understand the natural, endogenous role(s) of MSCs in normal and abnormal tissue functions. 
In conclusion, the present study revealed that hBMSC transplantation improved neuron regeneration in a rat model of MCAO, providing a possible therapeutic strategy for enhancing the nerve function of patients following stroke. However, due to the limited data and experimental environment, many improvements remain to be made in transplantation technology and experimental methods. It should also be noted that the study results may be affected by the limited sample size, and they should be further verified and observed in larger sample sizes. Additionally, the present study focused on exploring the function of MSCs without paying much attention to the regenerative power of MSCs, which might be studied in future research. In the future, research will be performed to uncover the typical mechanisms underlying the effect of hBMSC transplantation on neuron regeneration to provide a better and more effective treatment for neuronal dysfunction in patients after MCAO.

\section{Acknowledgements}

Not applicable.

\section{Funding}

The present study was supported by the Fundamental Research Funds for the Central Universities (grant no. 2042017kf0139), Innovative Seed Funds of Medical College of Wuhan University (grant no. TFZZ2018027), Natural Science Foundation of Hubei Province (grant no. ZRMS2019000278) and the National Natural Science Foundation of China (grant no. 81301052).

\section{Availability of data and materials}

The datasets used and/or analyzed during the current study are available from the corresponding author on reasonable request.

\section{Authors' contributions}

PX, MD, QGS and YGM wrote the paper, and conceived and designed the experiments. YZ and JHM analyzed the data. QC and SQL collected and provided the samples for the study. JQL, JC and FW obtained the results and conducted data analysis. JQL, JC and FW contributed to drafting the manuscript. All authors participated in the revised manuscript, and have read and approved the final submitted manuscript.

\section{Ethics approval and consent to participate}

The study was approved by the institutional review board of Renmin Hospital of Wuhan University. The experimental procedures and risks were announced to the subjects, and written informed consent was obtained from all participant. All animal experimentation in this study was approved by the Institutional Animal Care and Use Committee of Renmin Hospital of Wuhan University.

\section{Patient consent for publication}

Not applicable.

\section{Competing interests}

The authors declare that they have no competing interests.

\section{References}

1. Gibson CL: Cerebral ischemic stroke: Is gender important? J Cereb Blood Flow Metab 33: 1355-1361, 2013.

2. Fann DY, Lee SY, Manzanero S, Tang SC, Gelderblom M, Chunduri P, Bernreuther C, Glatzel M, Cheng YL, Thundyil J, et al: Intravenous immunoglobulin suppresses NLRP1 and NLRP3 inflammasome-mediated neuronal death in ischemic stroke. Cell Death Dis 4: e790, 2013.

3. Cho HY, Kim JS and Lee GC: Effects of motor imagery training on balance and gait abilities in post-stroke patients: A randomized controlled trial. Clin Rehabil 27: 675-680, 2013.

4. Metrot J, Mottet D, Hauret I, van Dokkum L, Bonnin-Koang HY, Torre $\mathrm{K}$ and Laffont I: Changes in bimanual coordination during the first 6 weeks after moderate hemiparetic stroke. Neurorehabil Neural Repair 27: 251-259, 2013.

5. Becker L and Mashimo H: Further promise of stem cells therapies in the enteric nervous system. Gastroenterology 136: 2055-2058, 2009.

6. Kulkarni S, Becker L and Pasricha PJ: Stem cell transplantation in neurodegenerative disorders of the gastrointestinal tract: Future or fiction? Gut 61: 613-621, 2012.

7. Garbossa D, Boido M, Fontanella M, Fronda C, Ducati A and Vercelli A: Recent therapeutic strategies for spinal cord injury treatment: Possible role of stem cells. Neurosurg Rev 35: D293-D311, 2012.

8. Maitland GD: The slump test: Examination and treatment. Aust J Physiother 31: 215-219, 1985.

9. Lin R, Ding Z, Ma H, Shi H, Gao Y, Qian W, Shi W, Sun Z, Hou $X$ and $\mathrm{Li} X$ : In vitro conditioned bone marrow-derived mesenchymal stem cells promote de novo functional enteric nerve regeneration, but not through direct-transdifferentiation. Stem Cells 33: 3545-3557, 2015.

10. Yamasaki S, Mera H, Itokazu M, Hashimoto Y and Wakitani S: Cartilage repair with autologous bone marrow mesenchymal stem cell transplantation: Review of preclinical and clinical studies. Cartilage 5: 196-200, 2014.

11. Chen L, Qu J and Xiang C: The multi-functional roles of menstrual blood-derived stem cells in regenerative medicine. Stem Cell Res Ther 10: 1, 2019.

12. Maxson S, Lopez EA, Yoo D, Danilkovitch-Miagkova A and Leroux MA: Concise review: Role of mesenchymal stem cells in wound repair. Stem Cells Transl Med 1: 142-149, 2012.

13. Tuan RS, Boland G and Tuli R: Adult mesenchymal stem cells and cell-based tissue engineering. Arthritis Res Ther 5: 32-45, 2003.

14. Pittenger MF, Mackay AM, Beck SC, Jaiswal RK, Douglas R, Mosca JD, Moorman MA, Simonetti DW, Craig S and Marshak DR: Multilineage potential of adult human mesenchymal stem cells. Science 284: 143-147, 1999.

15. Vanden Berg-Foels WS: In situ tissue regeneration: Chemoattractants for endogenous stem cell recruitment. Tissue Eng Part B Rev 20: 28-39, 2014.

16. Xu M, Stattin EL, Shaw G, Heinegard D, Sullivan G, Wilmut I, Colman A,Önnerfjord P, Khabut A, Aspberg A, et al: Chondrocytes derived from mesenchymal stromal cells and induced pluripotent cells of patients with familial osteochondritis dissecans exhibit an endoplasmic reticulum stress response and defective matrix assembly. Stem Cells Transl Med 5: 1171-1181, 2016.

17. Lindvall $\mathrm{O}$ and Kokaia Z: Stem cells in human neurodegenerative disorders--time for clinical translation? J Clin Invest 120: 29-40, 2010.

18. Scuteri A, Miloso M, Foudah D, Orciani M, Cavaletti G and Tredici G: Mesenchymal stem cells neuronal differentiation ability: A real perspective for nervous system repair? Curr Stem Cell Res Ther 6: 82-92, 2011.

19. Trzaska KA, King CC, Li KY, Kuzhikandathil EV, Nowycky MC, Ye JH and Rameshwar P: Brain-derived neurotrophic factor facilitates maturation of mesenchymal stem cell-derived dopamine progenitors to functional neurons. J Neurochem 110: 1058-1069, 2009

20. Greco SJ, Zhou C, Ye JH and Rameshwar P: An interdisciplinary approach and characterization of neuronal cells transdifferentiated from human mesenchymal stem cells. Stem Cells Dev 16: 811-826, 2007. 
21. Singer NG and Caplan AI: Mesenchymal stem cells: Mechanisms of inflammation. Annu Rev Pathol 6: 457-478, 2011.

22. Uccelli A, Moretta L and Pistoia V: Mesenchymal stem cells in health and disease. Nat Rev Immunol 8: 726-736, 2008.

23. Tohill M, Mantovani C, Wiberg M and Terenghi G: Rat bone marrow mesenchymal stem cells express glial markers and stimulate nerve regeneration. Neurosci Lett 362: 200-203, 2004.

24. Liu F and McCullough LD: Middle cerebral artery occlusion model in rodents: Methods and potential pitfalls. J Biomed Biotechnol 2011: 464701, 2011.

25. Zhang QW, Deng XX, Sun X, Xu JX and Sun FY: Exercise promotes axon regeneration of newborn striatonigral and corticonigral projection neurons in rats after ischemic stroke. PLoS One 8: e80139, 2013.

26. Bao X, Wei J, Feng M, Lu S, Li G, Dou W, Ma W, Ma S, An Y, Qin C, et al: Transplantation of human bone marrow-derived mesenchymal stem cells promotes behavioral recovery and endogenous neurogenesis after cerebral ischemia in rats. Brain Res 1367: 103-113, 2011.

27. He XY, Chen ZZ, Cai YQ, Xu G, Shang JH, Kou SB, Li M, Zhang HT, Duan CZ, Zhang SZ, et al: Expression of cytokines in rat brain with focal cerebral ischemia after grafting with bone marrow stromal cells and endothelial progenitor cells Cytotherapy 13: 46-53, 2011.

28. Mathivanan I, Trepp C, Brunold C, Baerlocher G and Enzmann V: Retinal differentiation of human bone marrow-derived stem cells by co-culture with retinal pigment epithelium in vitro. Exp Cell Res 333: 11-20, 2015.

29. Longa EZ, Weinstein PR, Carlson S and Cummins R: Reversible middle cerebral artery occlusion without craniectomy in rats. Stroke 20: 84-91, 1989.

30. McSweeney C and Mao Y: Applying stereotactic injection technique to study genetic effects on animal behaviors. J Vis Exp e52653,2015.

31. Chen J, Sanberg PR, Li Y, Wang L, Lu M, Willing AE, Sanchez-Ramos $\mathbf{J}$ and Chopp M: Intravenous administration of human umbilical cord blood reduces behavioral deficits after stroke in rats. Stroke 32: 2682-2688, 2001.

32. Honma T, Honmou O, Iihoshi S, Harada K, Houkin K, Hamada H and Kocsis JD: Intravenous infusion of immortalized human mesenchymal stem cells protects against injury in a cerebral ischemia model in adult rat. Exp Neurol 199: 56-66, 2006.

33. Hou M, Yang KM, Zhang H, Zhu WQ, Duan FJ, Wang H, Song YH, Wei YJ and Hu SS: Transplantation of mesenchymal stem cells from human bone marrow improves damaged heart function in rats. Int J Cardiol 115: 220-228, 2007.

34. Vital SA and Gavins FN: Surgical Approach for middle cerebral artery occlusion and reperfusion induced stroke in mice. J Vis Exp 2016

35. Wakabayashi K, Nagai A, Sheikh AM, Shiota Y, Narantuya D, Watanabe T, Masuda J, Kobayashi S, Kim SU and Yamaguchi S: Transplantation of human mesenchymal stem cells promotes functional improvement and increased expression of neurotrophic factors in a rat focal cerebral ischemia model. J Neurosci Res 88: 1017-1025, 2010.

36. Wei L, Fraser JL, Lu ZY, Hu X and Yu SP: Transplantation of hypoxia preconditioned bone marrow mesenchymal stem cells enhances angiogenesis and neurogenesis after cerebral ischemia in rats. Neurobiol Dis 46: 635-645, 2012.

37. Ma M, Ma Y, Yi X, Guo R, Zhu W, Fan X, Xu G, Frey WH 2nd and Liu X: Intranasal delivery of transforming growth factor-beta1 in mice after stroke reduces infarct volume and increases neurogenesis in the subventricular zone. BMC Neurosci 9: 117, 2008.

38. Chen J, Li Y, Wang L, Lu M, Zhang X and Chopp M: Therapeutic benefit of intracerebral transplantation of bone marrow stromal cells after cerebral ischemia in rats. J Neurol Sci 189: 49-57, 2001.

39. Cha HK, Cho HS and Choi JD: Effects of the nerve mobilization technique on lower limb function in patients with poststroke hemiparesis. J Phys Ther Sci 26: 981-983, 2014.

40. RolaR,Mizumatsu S, Otsuka S, Morhardt DR, Noble-Haeusslein LJ, Fishman K, Potts MB and Fike JR: Alterations in hippocampal neurogenesis following traumatic brain injury in mice. Exp Neurol 202: 189-199, 2006.
41. Ohnuma S and Harris WA: Neurogenesis and the cell cycle. Neuron 40: 199-208, 2003.

42. Alvarez-Buylla A and Lim DA: For the long run: Maintaining germinal niches in the adult brain. Neuron 41: 683-686, 2004.

43. Lindvall $O$ and Kokaia Z: Stem cells for the treatment of neurological disorders. Nature 441: 1094-1096, 2006.

44. Segal-Gavish H, Karvat G, Barak N, Barzilay R, Ganz J, Edry L, Aharony I, Offen D and Kimchi T: Mesenchymal stem cell transplantation promotes neurogenesis and ameliorates autism related behaviors in BTBR mice. Autism Res 9: 17-32, 2016.

45. Ishiwata T, Matsuda $Y$ and Naito Z: Nestin in gastrointestinal and other cancers: Effects on cells and tumor angiogenesis. World J Gastroenterol 17: 409-418, 2011.

46. Krylyshkina O, Chen J, Mebis L, Denef C and Vankelecom H: Nestin-immunoreactive cells in rat pituitary are neither hormonal nor typical folliculo-stellate cells. Endocrinology 146: 2376-2387, 2005.

47. Honda M, Tsuruta R, Kaneko T, Kasaoka S, Yagi T, Todani M, Fujita M, Izumi $\mathrm{T}$ and Maekawa T: Serum glial fibrillary acidic protein is a highly specific biomarker for traumatic brain injury in humans compared with S-100B and neuron-specific enolase. J Trauma 69: 104-109, 2010.

48. Lavezzi AM, Corna MF and Matturri L: Neuronal nuclear antigen (NeuN): A useful marker of neuronal immaturity in sudden unexplained perinatal death. J Neurol Sci 329: 45-50, 2013.

49. Gusel'nikova VV and Korzhevskiy DE: NeuN as a neuronal nuclear antigen and neuron differentiation marker. Acta Naturae 7: 42-47, 2015.

50. Kucia M, Zhang YP, Reca R, Wysoczynski M, Machalinski B, Majka M, Ildstad ST, Ratajczak J, Shields CB and Ratajczak MZ: Cells enriched in markers of neural tissue-committed stem cells reside in the bone marrow and are mobilized into the peripheral blood following stroke. Leukemia 20: 18-28, 2006.

51. Oh SH, Kim HN, Park HJ, Shin JY and Lee PH: Mesenchymal stem cells increase hippocampal neurogenesis and neuronal differentiation by enhancing the wnt signaling pathway in an Alzheimer's disease model. Cell Transplant 24: 1097-1109, 2015.

52. Shafei AE, Ali MA, Ghanem HG, Shehata AI, Abdelgawad AA, Handal HR, Talaat KA, Ashaal AE and El-Shal AS: Mesenchymal stem cell therapy: A promising cell-based therapy for treatment of myocardial infarction. J Gene Med 19: 2017.

53. Fiore EJ, Domínguez LM, Bayo J, García MG and Mazzolini GD: Taking advantage of the potential of mesenchymal stromal cells in liver regeneration: Cells and extracellular vesicles as therapeutic strategies. World J Gastroenterol 24: 2427-2440, 2018.

54. Meirelles Lda S, Fontes AM, Covas DT and Caplan AI: Mechanisms involved in the therapeutic properties of mesenchymal stem cells. Cytokine Growth Factor Rev 20: 419-427, 2009.

55. Karow M, Sánchez R, Schichor C, Masserdotti G, Ortega F, Heinrich C, Gascón S, Khan MA, Lie DC, Dellavalle A, et al: Reprogramming of pericyte-derived cells of the adult human brain into induced neuronal cells. Cell Stem Cell 11: 471-476, 2012.

56. Crisan M, Yap S, Casteilla L, Chen CW, Corselli M, Park TS, Andriolo G, Sun B, Zheng B, Zhang L, et al: A perivascular origin for mesenchymal stem cells in multiple human organs. Cell Stem Cell 3: 301-313, 2008.

This work is licensed under a Creative Commons Attribution-NonCommercial-NoDerivatives 4.0 International (CC BY-NC-ND 4.0) License. 